\title{
Consolidation Solutions of a Saturated Porothermoelastic Hollow Cylinder with Infinite Length
}

\section{Consolidation Solutions of Porothermoelastic Media}

\author{
Bing BAI \\ School of Civil Engineering, Beijing Jiaotong University, Beijing, China \\ Email: Baibing66@263.net \\ Received June 4, 2009; revised July 28, 2009; accepted August 10, 2009
}

\begin{abstract}
An analytical method is derived for the thermal consolidation of a saturated, porous, hollow cylinder with infinite length. The solutions in Laplace transform space are first obtained and then numerically inverted by Stehfest method. Two cases of boundary conditions are considered. First, variable thermal loadings are applied on the inner and outer pervious lateral surfaces of the hollow cylinder, and a variable mechanical loading with time is applied on the outer surface; while the displacement of the inner surface remains fixed. Secondly, variable thermal and mechanical loading are applied on the outer pervious surface, and the inner surface remains fixed, impervious and insulated. As two special problems, a solid cylinder with infinite length and a cylindrical cavity in a half-space body are also discussed. Finally, the evolutions of temperature, pore pressure and displacement with time along radial direction are analyzed by a numerical example.
\end{abstract}

Keywords: Porothermoelastic Media, Hollow Cylinder, Variable Thermal Loading, Consolidation Solutions, Stehfest Method

\section{Introduction}

The studies on the thermo-hydro-mechanical responses of saturated porous materials are widely used in various engineering fields such as the disposal of high-level nuclear waste, extraction of geothermal energy, storage of hot fluids, biomechanics to materials sciences, concrete resistance against fire, reliability of airfield.

There exists a substantial and growing literature to account for non-isothermal consolidation behavior of fluid-saturated porous materials [1-5]. Up to now, some analytical solutions to boundary and initial value problems have been developed under various scenarios. Booker and Savvidou [6] have presented solutions for the temperature, pressure and stress fields arising from a spherical heat source buried in a thermally consolidating material of infinite extent. McTigue [7] presented resolution methods and established exact solutions for a semiinfinite porous medium subjected to a constant surface temperature or heat flux with either drained or undrained boundary conditions. Smith and Booker [8] presented the Green's functions for a system of fully coupled linear equations governing thermal consolidation in a homogeneous isotropic material, and later gave a boundary integral method of numerical analysis. Giraud et al. [9] ana- lyzed the case of a heat source that decreases exponentially with time by considering a low-permeability clay for nuclear waste disposal. Wang and Papamichos $[10,11]$ discussed solutions for a cylindrical wellbore and a spherical cavity subjected to a constant temperature change and heat flow rate. Blond et al. [12] developed a closed-form solution for a porothermoelastic half-space submitted to a cyclic thermal loading, and a pressurediffusion equation that governs the fluctuation of the interstitial pressure was established. Bai [13] developed a solution approach for a planar thermal loading with variable intensity with time on the surface of a semiinfinite space. Bai [14] later derived an analytical method for the responses of saturated porous media subjected to cyclic thermal loading by using the Laplace transform and the Gauss-Legendre method of Laplace transform inversion. Abousleiman and Ekbote [15] presented the analytical solutions for an inclined hollow cylinder in a transversely isotropic material subjected to thermal and stress perturbations. Kanj et al. [16,17] applied an anisotropic porothermoelastic solution to an unjacketed hollow cylinder in a triaxial set-up. Bai [18] derived an analytical method for the thermal consolidation of layered, saturated porous half-space to variable thermal loading with time. 
In previous studies, the most concerned domains are the geometries such as a half-space body, a cylindrical cavity in a semi-infinite space and a cylindrical body, etc. In fact, hollow cylindrical geometries are also widely used in the laboratory for measurements of material properties and the understanding of the subsidence phenomena, formation consolidation and borehole stability. In this paper, analytical solutions for a hollow cylinder of porothermoelastic media with infinite length are derived aiming at experimental studies of the thermal consolidation of saturated porous materials such as soil that are carried out under non-isothermal conditions. The solutions in Laplace transform space are first obtained and then numerically inverted by Stehfest method. As two special problems, a solid cylinder with infinite length and a cylindrical cavity in a half-space body are also discussed. Based on the proposed solutions, numerical analyses are carried out to demonstrate the evolutions of temperature, pore pressure, displacement as well as radial and tangential stresses with time.

\section{Governing Equations}

For saturated, homogeneous, isotropic porous materials, the equilibrium equation of thermo-hydro-mechanical coupling consolidation may be written as $[8,10,12,14]$

$$
M \nabla^{2} \varepsilon_{v}-\alpha \nabla^{2} p-\beta \nabla^{2} \theta=0
$$

where $M=\lambda+2 G$ is the confined drained isothermal modulus; $\lambda$ and $G$ are Lamé constants; $\nabla^{2}$ is the Laplace operator; $\varepsilon_{\mathrm{v}}$ is the volumetric strain; $p$ is the excess pore pressure; $\theta=T-T_{0}$ is the increment of temperature above the ambient temperature, $T_{0}$ is the ambient absolute temperature, $T$ is the current absolute temperature; $\alpha=1-C_{\mathrm{s}} / C$ is Biot's coefficient, $C$ and $C_{\mathrm{s}}$ are the coefficients of volumetric compression of the solid skeleton and grains respectively; $\beta=3 \alpha_{\mathrm{s}} / C$ is the thermal expansion factor, and $\alpha_{\mathrm{s}}$ is the linear thermal expansion coefficient of solid grains.

According to Darcy's law and the continuity condition of seepage, the equation of mass conservation can be written as $[12,14]$

$$
\int_{0}^{t} \frac{k}{\gamma_{w}} \nabla^{2} p \mathrm{~d} t-\alpha \varepsilon_{v}+Y \theta-\alpha_{p} p=0
$$

where $k$ is the hydraulic conductivity; $\gamma_{\mathrm{w}}$ is the unit weight of pore water; $t$ is time; $\alpha_{\mathrm{p}}=n\left(C_{\mathrm{w}}-C_{\mathrm{s}}\right)+\alpha C_{\mathrm{s}}$; $Y=-3 n\left(\alpha_{\mathrm{w}}-\alpha_{\mathrm{s}}\right)-3 \alpha \alpha_{\mathrm{s}}, C_{\mathrm{w}}$ is the coefficient of volumetric compression of pore water; $\alpha_{\mathrm{w}}$ is the linear thermal expansion coefficient of pore water; and $n$ is the porosity of the medium.

According to Fourier's law of heat conduction, the equation of energy conservation can be written as $[12,14]$

$$
{ }_{0}^{t} \frac{K}{T_{0}} \nabla^{2} \theta \mathrm{d} t-Z \theta-\beta \varepsilon_{v}+Y p=0
$$

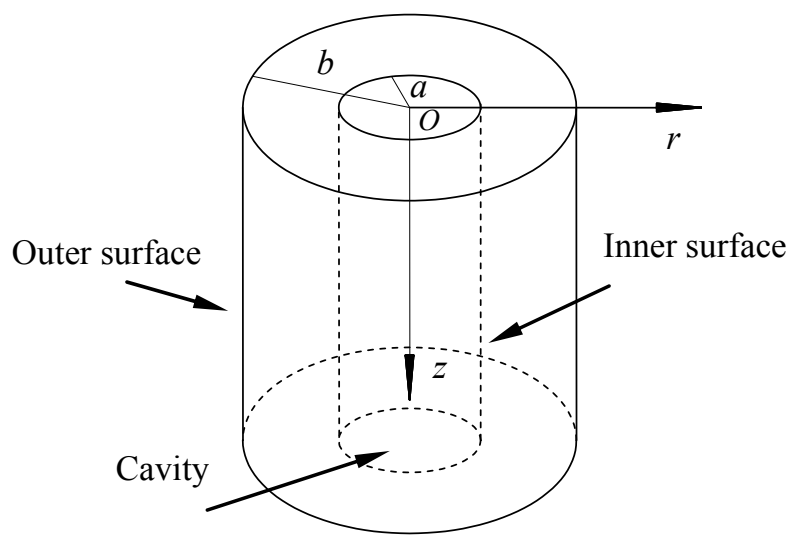

Figure 1. Mathematical model.

where $K$ is the coefficient of heat conductivity, $Z=[(1-n)$ $\left.\rho_{\mathrm{s}} c_{\mathrm{s}}+n \rho_{\mathrm{w}} c_{\mathrm{w}}\right] / T_{0}-3 \beta \alpha_{\mathrm{s}}$ is a coupling parameter, with $\rho_{\mathrm{w}}$ and $\rho_{\mathrm{s}}$ being the densities of pore water and solid grains, respectively, and $c_{\mathrm{w}}$ and $c_{\mathrm{s}}$ being the heat capacities of pore water and solid grains, respectively.

\section{Mathematical Model}

Consider the problem of a saturated, porous hollow cylinder with infinite length (see Figure 1). The following two cases of boundary conditions are imposed here.

Case 1: Variable thermal loading $\theta_{\mathrm{a}}(t)$ and $\theta_{\mathrm{b}}(t)$ are respectively applied on the inner and outer pervious lateral surfaces of the hollow cylinder. At the same time, a variable mechanical loading $p_{\mathrm{b}}(t)$ is also applied on the outer surface; while the displacement of the inner surface remains fixed. The origin $\mathrm{O}$ of the cylindrical coordinate system is selected at the center of the cylinder and the $z$-axis is the axis of rotational material symmetry, so that

$$
\begin{gathered}
\theta(a, t)=\theta_{a}(t) \cdot H(t), \quad p(a, t)=0, \quad u_{r}(a, t)=0 \quad(t>0) \\
\theta(b, t)=\theta_{b}(t) \cdot H(t), \quad p(b, t)=0, \quad \sigma_{r}(b, t)=p_{b}(t) \cdot H(t)
\end{gathered}
$$

where $a$ and $b$ are the inner and outer radius of the hollow cylinder, respectively; $\theta_{\mathrm{a}}(t)=T_{\mathrm{w} 1}(t)-T_{0}, \theta_{\mathrm{b}}(t)=T_{\mathrm{w} 2}(t)-$ $T_{0}$, with $T_{\mathrm{w} 1}(t)$ and $T_{\mathrm{w} 2}(t)$ are the current absolute temperatures of the inner and outer surfaces, respectively; $p_{\mathrm{b}}(t)$ is the mechanical loading of the outer surface; $u_{r}$ is the radial displacement; and $H(t)$ means the Heaviside unit step function.

Case 2: Variable thermal and mechanical loading $\theta_{b}(t)$ and $p_{\mathrm{b}}(t)$ are respectively applied on the outer pervious surface of the hollow cylinder. The inner surface remains fixed, impervious and insulated. Thus, the boundary conditions of the outer surface can still be expressed by Equation (5); while the boundary conditions of the inner surface are 


$$
\frac{\partial \theta(a, t)}{\partial r}=0, \frac{\partial p(a, t)}{\partial r}=0, u_{r}(a, t)=0
$$

It is noted that all applied boundary conditions on the cylinder may be time-dependent.

\section{Solution Approach}

\subsection{Solutions of the Governing Equations}

It is assumed that the initial conditions (Figure 1) are: $\theta(r$, $0)=0, p(r, 0)=0$, and $u_{\mathrm{r}}(r, 0)=0$. Then, upon Laplace transformation, Equations (1) to (3) become

$$
\begin{gathered}
M \nabla^{2} \bar{\varepsilon}_{v}-\alpha \nabla^{2} \bar{p}-\beta \nabla^{2} \bar{\theta}=0 \\
\frac{k}{\gamma_{w}} \nabla^{2} \bar{p}-\alpha s \bar{\varepsilon}_{v}+Y s \bar{\theta}-\alpha_{p} s \bar{p}=0 \\
\frac{K}{T_{0}} \nabla^{2} \bar{\theta}-Z s \bar{\theta}-\beta s \bar{\varepsilon}_{v}+Y s \bar{p}=0
\end{gathered}
$$

where $\nabla^{2}=\partial^{2} / \partial r^{2}+(\partial / \partial r) / r$, and $\bar{L}=\int_{0}^{\infty} e^{-s t} L \mathrm{~d} t$ $\left(L=\theta, p, \varepsilon_{\mathrm{v}}\right)$ and $s$ is the Laplace transform variable.

Equation (7) can be rewritten as

$$
\nabla^{2}\left[M \bar{\varepsilon}_{v}-\alpha \bar{p}-\beta \bar{\theta}\right]=0
$$

The integration of Equation (10) twice over $r$ yields

$$
M \bar{\varepsilon}_{v}-\alpha \bar{p}-\beta \bar{\theta}=h_{1}(s) \ln r+h_{2}(s)
$$

where $h_{1}$ and $h_{2}$ are arbitrary functions of $s$ to be determined from the boundary conditions.

From Equation (11), one has

$$
\bar{\varepsilon}_{v}=\frac{\alpha \bar{p}+\beta \bar{\theta}}{M}+D_{1} \ln r+D_{2}
$$

where $D_{1}=h_{1} / M, D_{2}=h_{2} / M$.

Substituting Equation (12) into Equations (8) and (9) results in, respectively

$$
\begin{aligned}
& a_{2} \nabla^{2} \bar{p}+a_{3} s \bar{\theta}+a_{4} s \bar{p}=\alpha\left(D_{1} \ln r+D_{2}\right) s \\
& b_{1} \nabla^{2} \bar{\theta}+b_{3} s \bar{\theta}+b_{4} s \bar{p}=\beta\left(D_{1} \ln r+D_{2}\right) s
\end{aligned}
$$

where $a_{2}=k / \gamma_{\mathrm{w}}, \quad a_{3}=Y-\alpha \beta / M, \quad a_{4}=-\alpha_{\mathrm{p}}-\alpha^{2} / M, \quad b_{1}=K / T_{0}$, $b_{3}=-Z-\beta^{2} / M, b_{4}=Y-\alpha \beta / M$.

Here, $c=-a_{2} / a_{4}=k /\left[\gamma_{\mathrm{w}}\left(\alpha_{\mathrm{p}}+\alpha^{2} / M\right)\right]$ is defined as the coefficient of thermal consolidation defined in previous work [8,11], $\kappa=-b_{1} / b_{3}=\left(K / T_{0}-\alpha_{\mathrm{w}} K_{\mathrm{w}} S_{\mathrm{w}}\right) /\left(Z+\beta^{2} / M\right)$ is defined as the thermal diffusivity. In fact, the ratio $c / \kappa$ reflects the relative rate of pore pressure dissipation to heat conductivity.

Using Equations (13) and (14), the elimination of term $\nabla^{2} \bar{\theta}$ leads to

$$
\bar{\theta}=\frac{f_{1}}{S} \nabla^{2} \bar{p}+f_{2} \bar{p}+f_{3}\left(D_{1} \ln r+D_{2}\right)
$$

where $f_{1}=-a_{2} / a_{3}, f_{2}=-a_{4} / a_{3}, f_{3}=\alpha / a_{3}$.

Substitution of Equation (15) into Equation (14) results in

$$
g_{1} \nabla^{4} \bar{p}+g_{2} s \nabla^{2} \bar{p}+g_{3} s^{2} \bar{p}+g_{4} s^{2}\left(D_{1} \ln r+D_{2}\right)=0(16)
$$

where $g_{1}=b_{1} f_{1}, g_{2}=b_{1} f_{2}+b_{3} f_{1}, g_{3}=b_{3} f_{2}+b_{4}, g_{4}=b_{3} f_{3}-\beta$.

It can be proved that the general solution of Equation (16) is

$$
\begin{gathered}
\bar{p}=A_{1} I_{0}(\sqrt{s \xi} r)+B_{1} K_{0}(\sqrt{s \xi} r)+A_{2} I_{0}(\sqrt{s \eta} r) \\
+B_{2} K_{0}(\sqrt{s \eta} r)-\frac{g_{4}}{g_{3}}\left(D_{1} \ln r+D_{2}\right)
\end{gathered}
$$

where $A_{1}, B_{1}, A_{2}$ and $B_{2}$ are arbitrary functions of $s$ to be determined from the boundary conditions, $I_{0}$ and $K_{0}$ are the modified first-kind and second-kind Bessel functions of order zero, respectively,

$$
\xi=\left(-g_{2}-\sqrt{g_{2}^{2}-4 g_{1} g_{3}}\right) /\left(2 g_{1}\right)
$$

and

$$
\eta=\left(-g_{2}+\sqrt{g_{2}^{2}-4 g_{1} g_{3}}\right) /\left(2 g_{1}\right) .
$$

Substitution of Equation (17) into Equation (15) results in

$$
\begin{gathered}
\bar{\theta}=\left(f_{1} \xi+f_{2}\right)\left[A_{1} I_{0}(\sqrt{s \xi} r)+B_{1} K_{0}(\sqrt{s \xi} r)\right]+ \\
\left(f_{1} \eta+f_{2}\right)\left[A_{2} I_{0}(\sqrt{s \eta} r)+B_{2} K_{0}(\sqrt{s \eta} r)\right] \\
+\left(f_{3}-f_{2} \frac{g_{4}}{g_{3}}\right)\left(D_{1} \ln r+D_{2}\right)
\end{gathered}
$$

Substitution of Equations (17) and (18) into Equation (12) results in

$$
\begin{gathered}
\bar{\varepsilon}_{v}=E_{1}\left[A_{1} I_{0}(\sqrt{s \xi} r)+B_{1} K_{0}(\sqrt{s \xi} r)\right] \\
+E_{2}\left[A_{2} I_{0}(\sqrt{s \eta} r)+B_{2} K_{0}(\sqrt{s \eta} r)\right]+E_{3}\left(D_{1} \ln r+D_{2}\right)
\end{gathered}
$$

where $E_{1}=\left[\alpha+\beta\left(f_{1} \xi+f_{2}\right)\right] / M, E_{2}=\left[\alpha+\beta\left(f_{1} \eta+f_{2}\right)\right] / M, E_{3}=$ $-\alpha g_{4} /\left(M g_{3}\right)+\beta\left(f_{3}-f_{2} g_{4} / g_{3}\right) / M+1$.

\subsection{Displacement, Stress and Strain}

Introduce a displacement potential function $\psi(r, t)$, then

$$
u_{r}=\frac{\partial \psi}{\partial r}
$$

Using Equation (19) and $\varepsilon_{\mathrm{v}}=\partial^{2} \psi / \partial r^{2}+(\partial \psi / \partial r) / r=\nabla^{2} \psi$, one has

$$
\begin{gathered}
\nabla^{2} \bar{\psi}=E_{1}\left[A_{1} I_{0}(\sqrt{s \xi} r)+B_{1} K_{0}(\sqrt{s \xi} r)\right] \\
+E_{2}\left[A_{2} I_{0}(\sqrt{s \eta} r)+B_{2} K_{0}(\sqrt{s \eta} r)\right]+E_{3}\left(D_{1} \ln r+D_{2}\right)
\end{gathered}
$$

The general solution of Equation (21) can be ex- 
pressed as

$$
\begin{gathered}
\bar{\psi}=\frac{E_{1}}{s \xi}\left[A_{1} I_{0}(\sqrt{s \xi} r)+B_{1} K_{0}(\sqrt{s \xi} r)\right] \\
+\frac{E_{2}}{s \eta}\left[A_{2} I_{0}(\sqrt{s \eta} r)+B_{2} K_{0}(\sqrt{s \eta} r)\right] \\
+\frac{E_{3}}{4}\left[D_{1} r^{2}(\ln r-1)+D_{2} r^{2}\right]+\left(D_{3} \ln r+D_{4}\right)
\end{gathered}
$$

where $D_{3}$ and $D_{4}$ are arbitrary functions of $s$ to be determined from the boundary conditions.

Using Equations (20) and (22) results in

$$
\begin{gathered}
\bar{u}_{r}=\frac{E_{1}}{\sqrt{s \xi}}\left[A_{1} I_{1}(\sqrt{s \xi} r)-B_{1} K_{1}(\sqrt{s \xi} r)\right] \\
+\frac{E_{2}}{\sqrt{s \eta}}\left[A_{2} I_{1}(\sqrt{s \eta} r)-B_{2} K_{1}(\sqrt{s \eta} r)\right] \\
+\frac{E_{3}}{4}\left[D_{1} r(2 \ln r-1)+2 D_{2} r\right]+\frac{D_{3}}{r}
\end{gathered}
$$

where $u_{\mathrm{r}}$ is radial displacement, $I_{1}$ and $K_{1}$ are the modified first-kind and second-kind Bessel functions of order one, respectively.

Using Equation (23) and generalized Hooke's law, radial stress $\sigma_{\mathrm{r}}$ and tangential stress $\sigma_{\varphi}$ can be obtained:

$$
\begin{gathered}
\bar{\sigma}_{r}=-2 G\left\{\frac{E_{1}}{\sqrt{s \xi} r}\left[A_{1} I_{1}(\sqrt{s \xi} r)-B_{1} K_{1}(\sqrt{s \xi} r)\right]\right. \\
+\frac{E_{2}}{\sqrt{s \eta} r}\left[A_{2} I_{1}(\sqrt{s \eta} r)-B_{2} K_{1}(\sqrt{s \eta} r)\right]+ \\
\left.\frac{E_{3}}{4}\left[D_{1}(2 \ln r-1)+2 D_{2}\right]+\frac{D_{3}}{r^{2}}\right\}-M D_{1} \ln r-M D_{2} \\
\bar{\sigma}_{\varphi}=-2 G\left\{E_{1} A_{1}\left[I_{0}(\sqrt{s \xi} r)-\frac{1}{\sqrt{s \xi} r} I_{1}(\sqrt{s \xi} r)\right]+\right. \\
E_{1} B_{1}\left[K_{0}(\sqrt{s \xi} r)+\frac{1}{\sqrt{s \xi} r} K_{1}(\sqrt{s \xi} r)\right] \\
E_{2} A_{2}\left[I_{0}(\sqrt{s \eta} r)-\frac{1}{\sqrt{s \eta} r} I_{1}(\sqrt{s \eta} r)\right]+ \\
E_{2} B_{2}\left[K_{0}(\sqrt{s \eta} r)+\frac{1}{\sqrt{s \eta}} K_{1}(\sqrt{s \eta} r)\right]+ \\
\left.\frac{E_{3}}{4}\left[D_{1}(2 \ln r+1)+2 D_{2}\right]-\frac{D_{3}}{r^{2}}\right\}-M D_{1} \ln r-M D_{2}
\end{gathered}
$$

\subsection{Inversion of the Laplace Transform}

Equations (17) to (19), (23) to (25) constitute the solutions in the Laplace transform domain. In reality, solutions in the real domain can all be obtained by inverting the above solutions. There are many numerical Laplace inversion schemes reported in literatures [10,17]. The Stehfest method has been extensively used, due to its accuracy, efficiency and stability. This method is based on sampling inversion data according to a delta series. The present study uses the Stehfest method.

\section{Determination of the Integration Functions}

In a cylindrical coordinate system, the following equilibrium is also used to determine the unknown coefficients in addition to the boundary conditions:

$$
\frac{\partial \sigma_{r}}{\partial r}+\frac{\sigma_{r}-\sigma_{\varphi}}{r}=0
$$

Substitution of Equations (24) and (25) into Equation (26) results in $D_{1}=0$. The remaining six arbitrary coefficients $\left(A_{1}, B_{1}, A_{2}, B_{2}, D_{2}\right.$ and $\left.D_{3}\right)$ can be determined by solving Equation (A9) (see Appendix A).

For the special case of $a \rightarrow 0$ (i.e. a solid cylinder with infinite length), there are three arbitrary coefficients $\left(A_{1}\right.$, $A_{2}$ and $D_{2}$ ), which can be determined by solving Equation (A10) (see Appendix B). For the special case of $b \rightarrow \infty$ (i.e. a cylindrical cavity in a half-space body), there are also three arbitrary coefficients $\left(B_{1}, B_{2}\right.$ and $\left.D_{3}\right)$, which can be determined by solving Equation (A11) (see Appendix C).

\section{Numerical Examples}

\subsection{Material Properties and Loading}

The material properties used in the analysis are given as follows: the elastic modulus $E=6.0 \times 10^{5} \mathrm{~Pa}$, the Poisson ratio $\mu=0.3$, the bulk modulus of solid grains $K_{\mathrm{s}}=2 \times 10^{10}$ $\mathrm{Pa}$, the bulk modulus of pore water $K_{\mathrm{w}}=5 \times 10^{9} \mathrm{~Pa}$, the thermal expansion coefficient of solid grains $\alpha_{\mathrm{s}}=$ $1.5 \times 10^{-5} /{ }^{\circ} \mathrm{C}$, the thermal expansion coefficient of pore water $\alpha_{\mathrm{w}}=2.0 \times 10^{-4} /{ }^{\circ} \mathrm{C}$, the porosity $n=0.4$, the heat capacity of solid grains $c_{\mathrm{s}}=800 \mathrm{~J} /\left(\mathrm{kg}{ }^{\circ} \mathrm{C}\right)$, the heat capacity of pore water $c_{\mathrm{w}}=4200 \mathrm{~J} /\left(\mathrm{kg}{ }^{\circ} \mathrm{C}\right)$, the density of pore water $\rho_{\mathrm{w}}=1.0 \times 10^{3} \mathrm{~kg} / \mathrm{m}^{3}$, the density of solid grains $\rho_{\mathrm{s}}$ $=2.6 \times 10^{3} \mathrm{~kg} / \mathrm{m}^{3}$, the coefficient of heat conductivity $K=0.5 \mathrm{~W} /\left(\mathrm{m}^{\circ} \mathrm{C}\right)$ and Biot's coefficient $\alpha=1.0$.

Geometrically, the hollow cylinder has an inner radius $a=0.02 \mathrm{~m}$; and an outer radius $b=0.08 \mathrm{~m}$ (see Figure 1 ). As such, the thickness of the cylinder wall $d=0.06 \mathrm{~m}$. For convenience, the following problem is discussed (i.e. Case 1): the thermal loading $\theta_{b}(t)$ and mechanical loading $p_{\mathrm{b}}(t)$ on the outer surface remain both constant; while an exponentially increasing temperature variation $\theta_{\mathrm{a}}(t)$ is applied on the inner surface. It can be written as

$$
\theta_{\mathrm{a}}(t)=\theta_{0}[1-\exp (-\omega t)]
$$

where $\theta_{0}=100^{\circ} \mathrm{C}, \omega=-0.00384 \mathrm{~s}^{-1}$. 


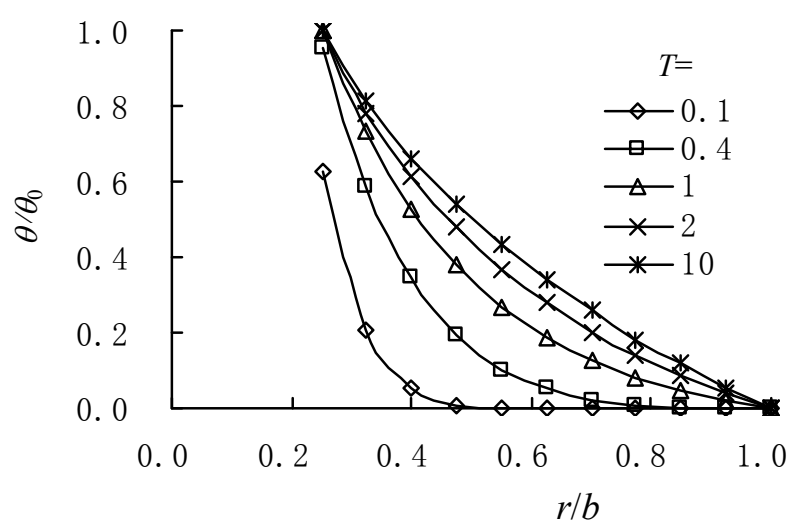

Figure 2. Distributions of temperature along radial distance.

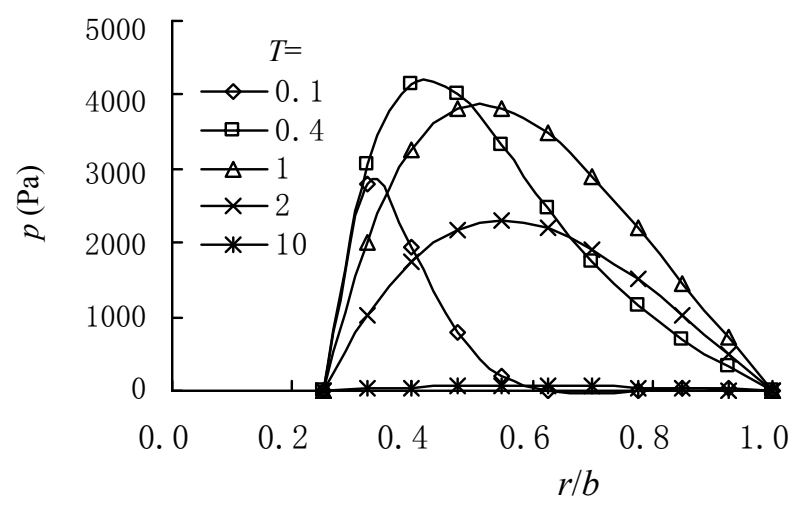

Figure 3. Pore pressure varying with $T$ under thermal loading.

\subsection{Responses under Thermal Loading}

In this section, we assume that the thermal loading $\theta_{\mathrm{b}}(t)=0$, and the mechanical loading $p_{\mathrm{b}}(t)=0$; while the thermal loading given by Equation (31) is applied on the inner surface. A dimensionless time is defined as $T=\kappa t / b^{2}$ (i.e. time factor). Figures 2 to 6 present respectively the temperature, pore pressure, radial displacement, radial stress and tangential stress distributions along radial distance for various time factors (e.g. $T=0.1,0.4,1,2,10$, i.e. $t=3.9,15.6,39.0,78.1,390.4 \mathrm{~min} ; c / \kappa=1)$.

It can be seen from Figure 2 that, with the elapsed time, the temperature is gradually conducted from the inner surface of the hollow cylinder to the points away from the surface. As the time factor $T$ increases continuously (e.g. $T=10$ ), the temperature values finally reach a quasi-steady state. At this time, the temperature distribution along the radial distance remains a steady temperature gradient. Certainly, the values of temperature gradient near the inner surface (e.g. $r / b=0.25-0.4$ ) are greater than those of the points in the vicinity of the outer surface (e.g. $r / b=0.8-1.0)$.

It can be seen from Figure 3 that at early times (e.g. $T=0.1,0.4$ ) the pore pressure value in the vicinity of the

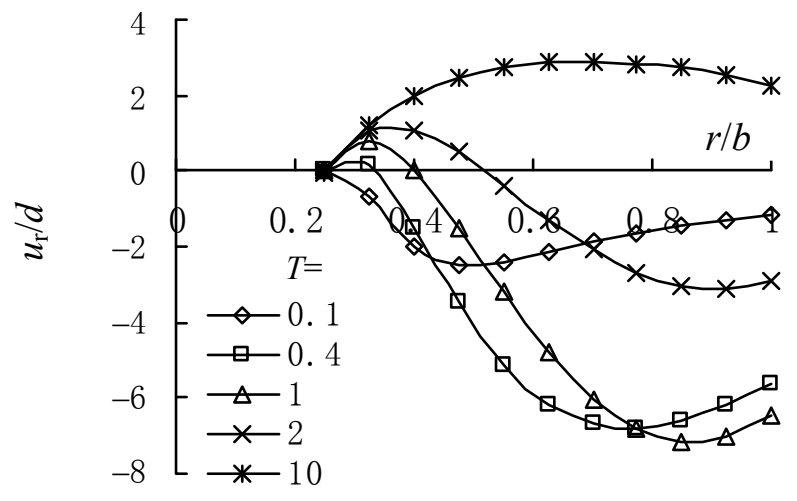

Figure 4. Radial displacement varying with $T$ under thermal loading.

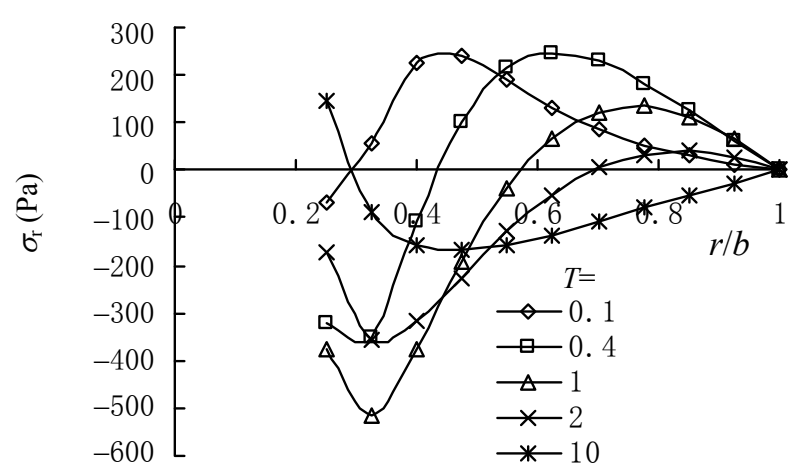

Figure 5. Radial stress varying with $T$ under thermal loading.

inner surface takes on a rising trend, and then is conducted from the inner surface of hollow cylinder to the outer surface; while the peak value gradually moves to the outer surface. At later times (e.g. $T=2,10)$, the pore pressure begins to decrease quickly due to the completely pervious lateral surfaces of the hollow cylinder, and finally is dissipated to zero along the radial distance.

Figure 4 shows that, at early times (e.g. $T=0.1$ ) the radial displacement takes on an expanding trend (i.e. negative displacement) along the whole radial distance; however with time factor $T$ increasing (e.g. $T=0.4,1,2$ ), the radial displacements of the points in the vicinity of the inner surface begin to contract (i.e. positive displacement) due to the strong coupling between the expansion of grains and the drainage of pore water. With the successive dissipation of pore pressure (e.g. $T=10$ ), the radial displacement eventually takes an increasing trend with the radial distance increasing.

As shown in Figures 5 and 6, in the whole processes of consolidation, the radial and tangential stress distributions are very complicated due to the coupling effects of pore pressure dissipation and thermal stress. In fact, at early times (e.g. $T=0.1,0.4,1)$ the radial stress in the vicinity of the inner surface begins to increase (being negative values, i.e. stretching stress), then (e.g. $T=2$ ) decreases with time factor increasing, and finally (e.g. 


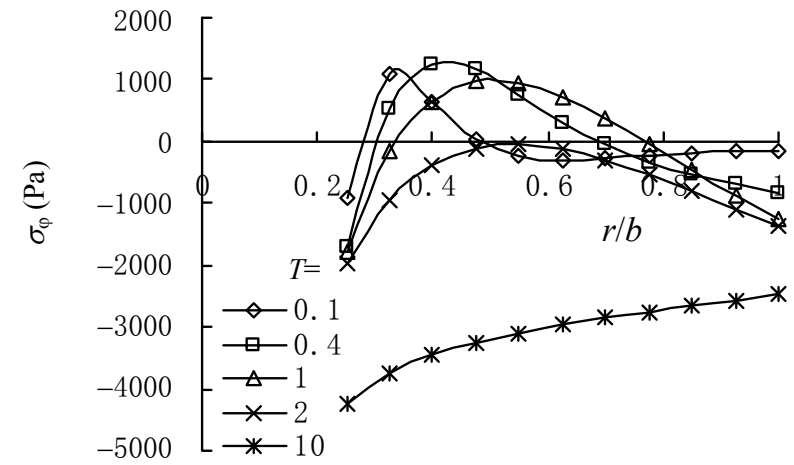

Figure 6. Tangential stress varying with $T$ under thermal loading.

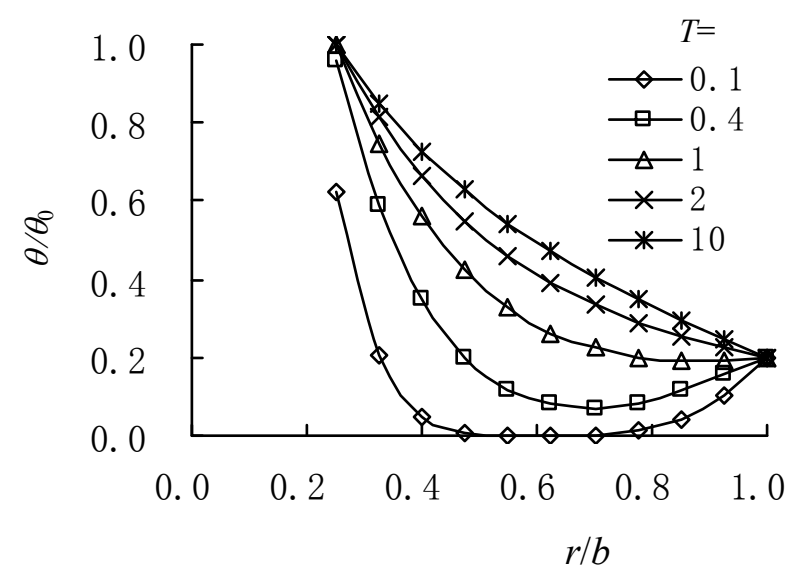

Figure 7. Distributions of temperature along radial distance $(c / k=1)$.

$T=10)$ takes on a positive value (i.e. compressive stress). On the other hand, the tangential stress in the middle part of the wall of the hollow cylinder initially takes a compressive state (i.e. positive value), and eventually presents a stretching state (i.e. negative value).

\subsection{Responses under Thermal and Mechanical Loading}

The responses of the porothermoelastic hollow cylinder under thermo-hydro-mechanical coupling are discussed in this section. Here, the thermal loading $\theta_{\mathrm{b}}(t)=20^{\circ} \mathrm{C}$, and the mechanical loading $p_{\mathrm{b}}(t)=100 \mathrm{kPa}$; while the thermal loading given by Equation (31) is applied on the inner surface. Figures 7 to 11 present respectively the temperature, pore pressure, radial displacement, radial stress and tangential stress distributions as a function of the radial distance for various time factors (e.g. $T=0.1,0.4,1$, $2,10 ; c / \kappa=1)$.

It can be seen from Figure 7 that the developing trend of the temperature along the radial distance is similar to the temperature distributions in Figure 2 except for the values on the outer surface of the hollow cylinder, which

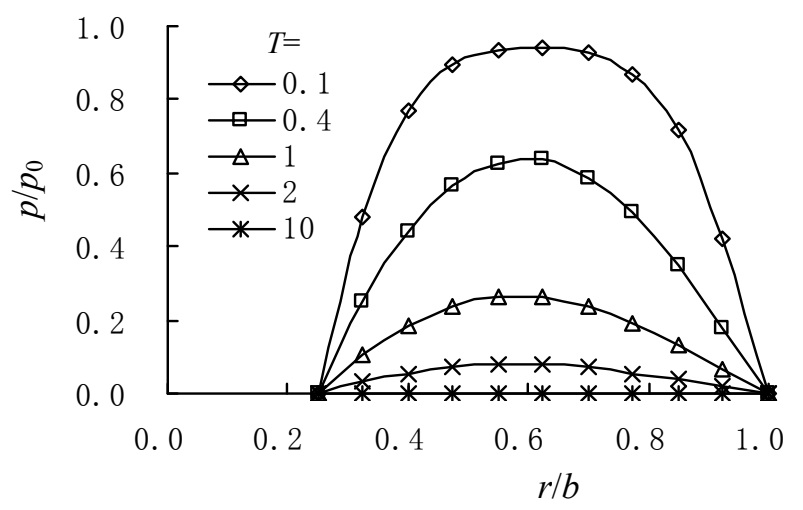

Figure 8. Distributions of pore pressure along radial distance $(c / k=1)$.

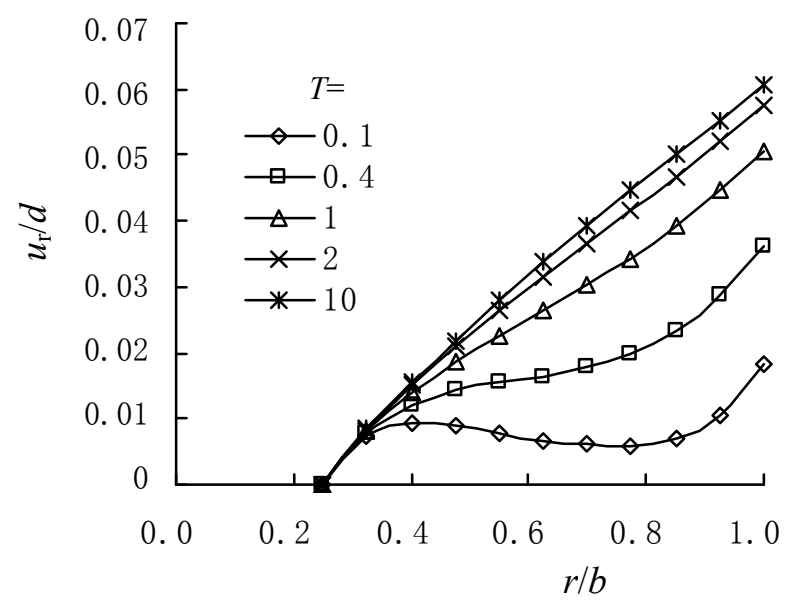

Figure 9. Distributions of radial displacement along radial distance $(c / k=1)$.

is due to the difference of boundary thermal loading. Calculation results for various $c / \kappa$ (e.g. $c / \kappa=0.1,1,2$ ) show that the coupling effects of displacement and stress fields on temperature field can be generally neglected.

By virtue of the imposed lateral boundary conditions, the pore pressure drops almost instantaneously at the inner and outer boundaries (i.e. $r=a$ and $r=b$ ) as indicated in Figure 8. As such, there exists a peak value of pore pressure in the inner layers. As time progresses, the pore pressure peak value gradually diffuses and flattens. It should be noted that the pore pressure in the vicinity of the outer boundary seems to dissipate more quickly, which is due to the greater drainage surface of the outer boundary than that of the inner boundary.

Figure 9 shows that the radial displacement anywhere in the cylinder contracts (i.e. being positive value) with the diffusion of the pore water. However, it is noticed that, at early times (e.g. $T=0.1,0.4$ ), the radial displacement in the local range of the wall (e.g. $0.4<r / b<0.9$ ) is even smaller than that in the vicinity of the inner boundary (here, noting $\left.u_{\mathrm{r}}(a, t)=0\right)$. This may be attributed to the 


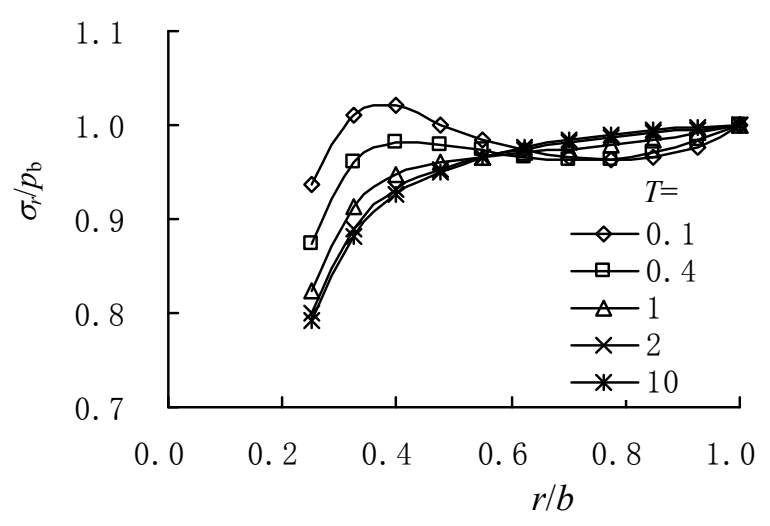

Figure 10. Distributions of radial stresses along radial distance $(c / k=1)$.

consolidation deformation (i.e. shrinkage of the cylinder) caused by the rapid dissipation in the vicinity of the pervious inner and outer surfaces. Obviously, this phenomenon vanishes as time progresses (see Figure 9). Furthermore, the radial displacement $u_{\mathrm{r}}$ will take on a linear relation with radial distance $r$ at time factor $T$ tends to infinity.

It can be seen from Figure 10 that the evolution of radial stress is very complicated. At early times (e.g. $T=0.1,0.4$ ), the radial stress in the local range of the wall (e.g. $0.3<r / b<0.4$ ) is even greater than the applied mechanical loading $p_{\mathrm{b}}$ on the outer surface (i.e. $\sigma_{\mathrm{r}} / p_{\mathrm{b}}>1$ ). Obviously, as time factor $T$ increases, the radial stress takes on a monotonically increasing trend with radial distance, and finally reach a steady state at time factor $T$ tends to infinity.

Figure 11 presents a tangential stress concentration at each of the boundaries of the cylinder. These stresses are generated as a result of the hoop effects that accompany the inner and outer diffusion fronts. Moreover, this stress concentration is more severe at early times. With diffusion, the tangential stress concentrations at the lateral surfaces weaken and diminish in magnitude while higher compressive tangential stresses $\left(\sigma_{\phi} / p_{\mathrm{b}}>1\right)$ are noted to form inside the cylinder.

\section{Conclusions}

1) An analytical method is derived for the thermal consolidation of a saturated, porous, hollow cylinder with infinite length. The solutions in Laplace transform space are first obtained and then numerically inverted by Stehfest method. As two special problems, a solid cylinder with infinite length and a cylindrical cavity in a half-space body are also discussed.

2) The responses of a porothermoelastic hollow cylinder subjected to exponentially increasing thermal loading with time on the inner surface are discussed. Calculations show that, as the temperature is gradually conducted from the inner surface to the points away from the surface,

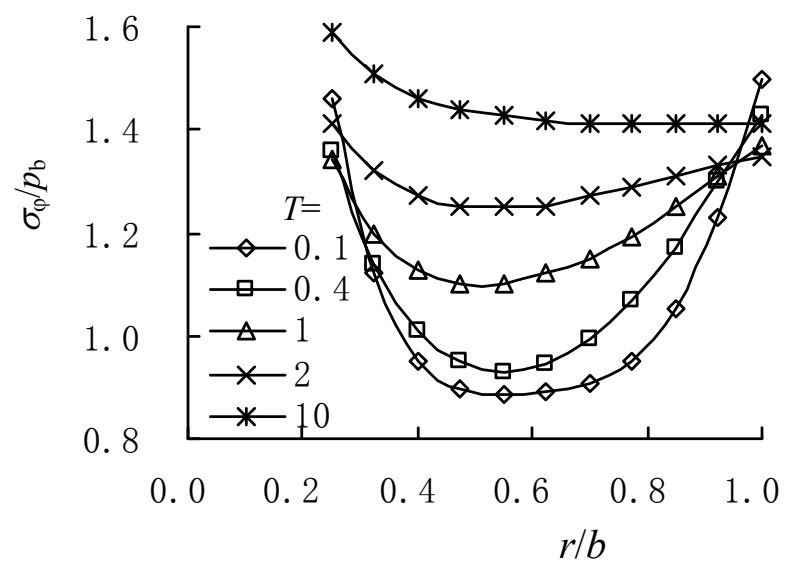

Figure 11. Distributions of tangential stresses along radial distance $(c / k=1)$.

the pore pressure value initially takes on a rising trend, and then begins to decrease quickly due to the pervious lateral surfaces. In addition, the radial displacement initially increases with the increase of temperature and then contracts with the diffusion of pore water, which is due to the strong coupling effects of the expansion of grains, the drainage of pore water, and the radial and tangential stress varying with time under thermal loading.

3) The responses of a porothermoelastic hollow cylinder under thermo-hydro-mechanical coupling have also been analyzed. Numerical results indicate that the temperature difference generates both pore pressure and stress distributions in the cylinder. A pore pressure field, as well as a radial displacement, a radial and tangential stress fields, are created as a result of the compaction of the cylinder and the heating of the borehole wall. Correspondingly, this results in the complicated evolution processes of all the variables, and can be explained by the consolidation deformation due to the thermal stress and the rapid dissipation of pore pressure in the vicinity of the pervious inner and outer surfaces.

\section{Acknowledgements}

Financial support from the National Natural Science Foundation of China (NSFC) under approved grant No. 50879003 is gratefully acknowledged.

\section{References}

[1] M. Kurashige, "A thermoelastic theory of fluid-filled porous materials," International Journal of Solids and Structures, Vol. 25, No. 9, pp. 1039-1052, 1989.

[2] X. Li, "A generalized theory of thermoelasticity for an anisotropic medium," International Journal of Engineering Science, Vol. 30, No. 5, pp. 571-577, 1992.

[3] H. N. Seneviratne, J. P. Carter, D. W. Airey, and J. R. Booker, "A review of models for predicting the ther- 
momechanical behavior of soft clays," International Journal for Numerical and Analytical Methods in Geomechanics, Vol. 17, No. 10, pp. 715-733,1993.

[4] M. Bai and Y. Abousleiman, "Thermoporoelastic coupling with application to consolidation," International Journal for Numerical and Analytical Methods in Geomechanics, Vol. 21, No. 2, pp. 121-132, 1997.

[5] R. W. Zimmerman, "Coupling in poroelasticity and thermoelasticity," International Journal of Rock Mechanics and Mining Sciences, Vol. 37, No. 1, pp. 79-87, 2000.

[6] J. R. Booker and C. Savvidou, "Consolidation around a spherical heat source," International Journal of Solids and Structures, Vol. 20, No. 5, pp. 1079-1090, 1984.

[7] D. F. McTigue, "Flow to a heated borehole in porous, thermoelastic rock: Analysis," Water Resources Research, Vol. 26, No. 8, pp. 1763-1774, 1990.

[8] D. W. Smith and J. R. Booker, "Green's function for a fully coupled thermoporoelastic materials," International Journal for Numerical and Analytical Methods in Geomechanics, Vol. 17, No. 2, pp. 139-163, 1993.

[9] A. Giraud, F. Homand, and G. Rousset, "Thermoelastic and thermoplastic response of a double-layer porous space containing a decaying heat source," International Journal for Numerical and Analytical Methods in Geomechanics, Vol. 22, No. 2, pp. 133-149, 1998.

[10] Y. Wang and E. Papamichos, "An analytical solution for conductive heat flow and the thermally induced fluid flow around a wellbore in a poroelastic medium," Water Resource Research, Vol. 36, No. 5, pp. 3375-3384, 1994.

[11] Y. Wang and E. Papamichos, "Thermal effects on fluid flow and hydraulic fracturing from wellbores and cavities in low-permeability formations," International Journal for Numerical and Analytical Methods in Geomechanics, Vol. 23, No. 15, pp. 1819-1834, 1999.

[12] E. Blond, N. Schmitt, and F. Hild, "Response of saturated porous media to cyclic thermal loading," International Journal for Numerical and Analytical Methods in Geomechanics, Vol. 27, No. 11, pp. 883-904, 2003.

[13] B. Bai, "Response of saturated porous media subjected to local thermal loading on the surface of semi-infinite space," Acta Mechanica Sinica, Vol. 22, No. 1, pp. 54-61, 2006.

[14] B. Bai, "Fluctuation responses of saturated porous media subjected to cyclic thermal loading," Computers and Geotechnics, Vol. 33, No. 4, pp. 396-403, 2006.

[15] Y. Abousleiman and S. Ekbote, "Solutions for the inclined borehole in a porothermoelastic transversely isotropic medium," Journal of applied Mechanics, Vol. 72, No. 2, pp. 102-114, 2005.

[16] M. Kanj, Y. Abousleiman, and R. Ghanem, "Poromechanics of anisotropic hollow cylinders," Journal of Engineering Mechanics, Vol. 129, No. 11, pp. 1277-1287, 2003.

[17] M. Kanj and Y. Abousleiman, "Porothermoelastic analyses of anisotropic hollow cylinders with applications," International Journal for Numerical and Analytical Methods in Geomechanics, Vol. 29, No. 1, pp. 103-126, 2005.

[18] B. Bai, "Thermal consolidation of layered porous halfspace to variable thermal loading," Applied Mathematics and Mechanics, Vol. 27, No. 11, pp. 1531-1539, 2006. 


\section{Appendix A}

For the first case of boundary conditions (i.e. Case 1), from Equations (4) and (5), when $t>0, \bar{\theta}(a, t)=\bar{\theta}_{a}$, $\bar{p}(a, t)=0, \bar{u}_{r}(a, t)=0, \quad \bar{\theta}(b, t)=\bar{\theta}_{b}, \quad \bar{p}(b, t)=0 \quad$ and $\bar{\sigma}_{r}(b, t)=\bar{p}_{b}$, which yields

$$
\begin{gathered}
A_{1} r I_{0}(\sqrt{s \xi} a)+B_{1} r K_{0}(\sqrt{s \xi} a)+A_{2} q I_{0}(\sqrt{s \eta} a) \\
+B_{2} q K_{0}(\sqrt{s \eta} a)+D_{2} v=\bar{\theta}_{a} \\
A_{1} I_{0}(\sqrt{s \xi} a)+B_{1} K_{0}(\sqrt{s \xi} a)+A_{2} I_{0}(\sqrt{s \eta} a) \\
+B_{2} K_{0}(\sqrt{s \eta} a)+D_{2} u=0 \\
A_{1} \frac{E_{1}}{\sqrt{s \xi}} I_{1}(\sqrt{s \xi} a)-B_{1} \frac{E_{1}}{\sqrt{s \xi}} K_{1}(\sqrt{s \xi} a) \\
+A_{2} \frac{E_{2}}{\sqrt{s \eta}} I_{1}(\sqrt{s \eta} a)-B_{2} \frac{E_{2}}{\sqrt{s \eta}} K_{1}(\sqrt{s \eta} a) \\
+D_{2} \frac{E_{3} a}{2}+\frac{D_{3}}{a}=0 \\
A_{1} r I_{0}(\sqrt{s \xi} b)+B_{1} r K_{0}(\sqrt{s \xi} b)+A_{2} q I_{0}(\sqrt{s \eta} b) \\
+B_{2} q K_{0}(\sqrt{s \eta} b)+D_{2} v=\bar{\theta}_{b} \\
A_{1} I_{0}(\sqrt{s \xi} b)+B_{1} K_{0}(\sqrt{s \xi} b)+A_{2} I_{0}(\sqrt{s \eta} b) \\
+B_{2} K_{0}(\sqrt{s \eta} b)+D_{2} u=0 \\
A_{1} \frac{-2 G E_{1}}{\sqrt{s \xi} b} I_{1}(\sqrt{s \xi} b)+B_{1} \frac{2 G E_{1}}{\sqrt{s \xi} b} K_{1}(\sqrt{s \xi} b) \\
+A_{2} \frac{-2 G E_{2}}{\sqrt{s \eta} b} I_{1}(\sqrt{s \eta} b)+B_{2} \frac{2 G E_{2}}{\sqrt{s \eta} b} K_{1}(\sqrt{s \eta} b) \\
+D_{2} w-D_{3} \frac{2 G}{b^{2}}=\bar{p}_{b}
\end{gathered}
$$

where $r=f_{1} \xi+f_{2}, \quad q=f_{1} \eta+f_{2}, \quad u=-g_{4} / g_{3}$, $v=f_{3}-f_{2} g_{4} / g_{3}, w=-M-G E_{3}$.

For the second case of boundary conditions (i.e. Case 2), from $\bar{u}_{r}(a, t)=0$ and Equation (5), the Equations (A3) to (A6) can be obtained easily; while using Equation (6), when $t>0, \partial \bar{\theta}(a, t) / \partial r=0$ and $\partial \bar{p}(a, t) / \partial r=0$, which yields

$$
\begin{gathered}
A_{1} r \sqrt{s \xi} I_{1}(\sqrt{s \xi} a) \\
-B_{1} r \sqrt{s \xi} K_{1}(\sqrt{s \xi} a) \\
+A_{2} q \sqrt{s \eta} I_{1}(\sqrt{s \eta} a) \\
-B_{2} q \sqrt{s \eta} K_{1}(\sqrt{s \eta} a)=0 \\
A_{1} \sqrt{s \xi} I_{1}(\sqrt{s \xi} a)-B_{1} \sqrt{s \xi} K_{1}(\sqrt{s \xi} a)+A_{2} \sqrt{s \eta} I_{1}(\sqrt{s \eta} a) \\
\left.-B_{2} \sqrt{s \eta} K_{1}(\sqrt{s \eta} a)=0 \quad \text { (A }\right)
\end{gathered}
$$

Hence, the following equation is obtained:

$$
\left[\begin{array}{llllll}
d_{11} & d_{12} & d_{13} & d_{14} & d_{15} & d_{16} \\
d_{21} & d_{22} & d_{23} & d_{24} & d_{25} & d_{26} \\
d_{31} & d_{32} & d_{33} & d_{34} & d_{35} & d_{36} \\
d_{41} & d_{42} & d_{43} & d_{44} & d_{45} & d_{46} \\
d_{51} & d_{52} & d_{53} & d_{54} & d_{55} & d_{56} \\
d_{61} & d_{62} & d_{63} & d_{64} & d_{65} & d_{66}
\end{array}\right]\left[\begin{array}{l}
A_{1} \\
B_{1} \\
A_{2} \\
B_{2} \\
D_{2} \\
D_{3}
\end{array}\right]=\left[\begin{array}{l}
\bar{\theta}_{a} \text { or } 0 \\
0 \\
0 \\
\bar{\theta}_{b} \\
0 \\
\bar{p}_{b}
\end{array}\right]
$$

where the coefficients $d_{11}, d_{12}, \ldots, d_{65}$ and $d_{66}$ can be given correspondingly by Equations (A1) to (A6) (for Case 1) or Equations (A7), (A8) and (A3) to (A6) (for Case 2).

\section{Appendix B}

Due to $K_{1}(\sqrt{s \xi} a)=\infty, \quad K_{1}(\sqrt{s \eta} a)=\infty$ and $1 / a=\infty$ at $a \rightarrow 0$ in Equations (A7), (A8) and (A3), one has $B_{1}=0$, $B_{2}=0$ and $D_{3}=0$. This implies that all variables must be finite. Hence, Equations (A7), (A8) and (A3) are satisfied automatically (noting $I_{1}(0)=0$ ). The remaining three arbitrary coefficients $\left(A_{1}, A_{2}\right.$ and $\left.D_{2}\right)$ can be determined by solving Equtions (A4) to (A6) simultaneously. At this time, Equation (A9) reduces to the following expression:

$$
\left[\begin{array}{lll}
d_{41} & d_{43} & d_{45} \\
d_{51} & d_{53} & d_{55} \\
d_{61} & d_{63} & d_{65}
\end{array}\right]\left[\begin{array}{l}
A_{1} \\
A_{2} \\
D_{2}
\end{array}\right]=\left[\begin{array}{l}
\bar{\theta}_{b} \\
0 \\
\bar{p}_{b}
\end{array}\right]
$$

where the coefficients $d_{41}, d_{43}, \ldots, d_{63}$ and $d_{65}$ can be given correspondingly by Equations (A4) to (A6).

\section{Appendix C}

Due to $I_{0}(\sqrt{s \xi} b)=\infty$ and $I_{0}(\sqrt{s \eta} b)=\infty$ at $b \rightarrow \infty$ in Equations (A4) to (A6), one has $A_{1}=0$ and $A_{2}=0$. Noting $K_{0}(\infty)=0$ and $K_{1}(\infty)=0$, for the satisfaction of Equations (A4) to (A6), one must let $D_{2}=0$. The remaining three arbitrary coefficients $\left(B_{1}, B_{2}\right.$ and $\left.D_{3}\right)$ can be determined by solving Equations (A1) to (A3) simultaneously. At this time, Equation (A9) reduces to the following expression:

$$
\left[\begin{array}{lll}
d_{12} & d_{14} & d_{16} \\
d_{22} & d_{24} & d_{26} \\
d_{32} & d_{34} & d_{36}
\end{array}\right]\left[\begin{array}{l}
B_{1} \\
B_{2} \\
D_{3}
\end{array}\right]=\left[\begin{array}{l}
\bar{\theta}_{a} \\
0 \\
0
\end{array}\right]
$$

where the coefficients $d_{12}, d_{14}, \ldots, d_{34}$ and $d_{36}$ can be given correspondingly by Equations (A1) to (A3). 